\title{
Consumo de anticoncepcionais e fatores associados entre estudantes universitários
}

\section{Contraceptive use and associated factors among university students}

\author{
Gabriel Silvério de Souza ${ }^{1}$, Camila Cristina Lunardelli Zanfrilli², \\ Cecília Valério Martins ${ }^{2}$, Pedro Eduardo Horácio Cleto (in memoriam) ${ }^{2}$, \\ Camilo Molino Guidoni ${ }^{3}$, Edmarlon Girotto ${ }^{4}$
}

Resumo

\begin{abstract}
Introdução: o uso de anticoncepcionais vem crescendo a cada ano, sendo um dos principais motivos para a redução das taxas de fecundidade total, inclusive na população mais jovem, como a universitária. Objetivo: identificar o consumo de anticoncepcionais hormonais e fatores associados entre estudantes universitárias. Métodos: a população de estudo foi composta por estudantes universitárias matriculadas nos cursos de graduação da Universidade Estadual de Londrina (UEL), em Londrina, estado do Paraná, Brasil, no ano de 2019, integrantes do projeto maior denominado GraduaUEL. As estudantes responderam um instrumento construído em uma plataforma digital on-line. A variável dependente foi o consumo de contraceptivos hormonais, e as variáveis independentes foram aspectos sociodemográficos e hábitos de vida e de saúde. As associações medidas foram verificadas por meio de Regressão de Poisson, com cálculo da Razão de Prevalência (RP) e intervalo de confiança de 95\% (IC 95\%). Resultados: das 2.221 estudantes avaliadas, identificou-se o consumo de contraceptivos por $13,0 \%$, maior entre mulheres brancas/amarelas, que relataram serem heterossexuais, que apresentavam pais com maior escolaridade, que referiram possuir plano privado de saúde e companheiro, e que praticavam atividade física pelo menos duas vezes na semana. Entretanto, apenas o fato de serem heterossexuais mostrou-se associação significativa após a análise ajustada (RP: 1,865; IC 95\%: 1,308-2,659). Conclusões: diante do exposto, fica clara a necessidade de preparar os profissionais de saúde sobre os contraceptivos e seus efeitos adversos; ainda, que questões sociais devem ser consideradas no processo de uso dos contraceptivos, para combater a discriminação e as desigualdades social e econômica quanto ao acesso e uso destes medicamentos.
\end{abstract}

Palavras-chave: Contraceptivos hormonais; Estudantes; Ensino superior; Uso racional de medicamentos.

\footnotetext{
${ }^{1}$ Graduando em Farmácia na Universidade Estadual de Londrina (UEL), Londrina, Paraná, Brasil. E-mail: gabriel.silverio@uel.br

${ }^{2}$ Graduandos em Farmácia na Universidade Estadual de Londrina, Londrina, Paraná, Brasil.

${ }^{3}$ Doutorado em Ciências Farmacêuticas (Medicamentos e Cosméticos) pela Faculdade de Ciências Farmacêuticas de Ribeirão Preto da Universidade de São Paulo (FCFRP-USP), Ribeirão Preto, São Paulo, Brasil. Professor no Departamento de Ciências Farmacêuticas do Centro de Ciências da Saúde da Universidade Estadual de Londrina, Londrina, Paraná, Brasil. Coordenador do Centro de Informações e Assistência Toxicológicas (CIATox) do Hospital Universitário da Universidade Estadual de Londrina (HU-UEL), Londrina, Paraná, Brasil.

${ }^{4}$ Doutorado em Saúde Coletiva pela Universidade Estadual de Londrina, Londrina, Paraná, Brasil. Professor no Departamento de Ciências Farmacêuticas do Centro de Ciências da Saúde da Universidade Estadual de Londrina, Londrina, Paraná, Brasil. Diretor da Farmácia Universitária da Universidade Estadual de Londrina, Londrina, Paraná, Brasil.
} 


\begin{abstract}
Introduction: the use of contraceptives has been growing every year, being one of the main reasons for the reduction in total fertility rates, including in the younger population, such as university students. Objective: identify the consumption of hormonal contraceptives and associated factors among university students. Methods: the study population consisted of university students enrolled in undergraduate courses at the Universidade Estadual de Londrina (UEL), in Londrina, Paraná state, Brazil, in 2019, members of the larger project called GraduaUEL. The students answered an instrument built on an on-line digital platform. The dependent variable was the consumption of hormonal contraceptives, and the independent variables were sociodemographic aspects, lifestyle and health. Measured associations were verified using Poisson Regression, with calculation of the Prevalence Ratio (PR) and 95\% confidence interval $(95 \% \mathrm{CI})$. Results: of the 2,221 students evaluated, the consumption of contraceptives was identified by $13.0 \%$, higher among white/yellow women, who reported being heterosexual, who had parents with higher education, who reported having a private health plan and partner, and who practiced physical activity at least twice a week. However, only the fact of being heterosexual showed a significant association after the adjusted analysis (PR: 1.865; 95\% CI: 1.308-2.659). Conclusions: in light of the above, the need to prepare health professionals about contraceptives and their adverse effects is clear, as well as what social issues should be considered in the process of using contraceptives, to combat discrimination, social and economic inequality regarding access and the use of these medications.
\end{abstract}

Keywords: Hormonal contraceptive agents; Students; Higher education; Rational use of medicines.

\section{Introdução}

Nos últimos anos, as populações jovem e adulta brasileira têm diminuído proporcionalmente à população idosa. Segundo o Instituto Brasileiro de Geografia e Estatística (IBGE), no ano de 2030 o perfil demográfico da população tenderá a ter mais idosos do que crianças e jovens; no ano de 2047 esse crescimento estacionará. ${ }^{(1)}$ Isso se deve muito à redução da fecundidade que se tem observado no Brasil. Entre os anos de 2010 e 2015, as taxas de fecundidade total (número médio de filhos nascidos vivos, tidos por mulher ao final do seu período reprodutivo) reduziram de 1,87 para 1,72 no País, ou seja, queda de $8,0 \%{ }^{(2)}$ Logo, espera-se que haja uma diminuição ainda maior nos próximos anos.

A redução da fecundidade deve-se a vários fatores, entre eles, os jovens das gerações $\mathrm{Y}$ e Z tendem a querer se autorrealizar, ou seja, realizar primeiramente seus objetivos, como profissão e ter estabilidade financeira, para depois pensarem em uma possibilidade de ter um filho; outra explicação seria das incertezas de suas vidas no futuro, assim como o aumento da taxa de desemprego que os anseiam. ${ }^{(3-4)}$
Nesse contexto, o consumo de contraceptivos tem crescido nas últimas décadas, inclusive no Brasil. No ano de 2015, relatório da Organização das Nações Unidas (ONU) apontou que 79,0\% das brasileiras de 15 a 49 anos casadas ou em união estável faziam uso de contraceptivos, comparados a 51,0\% na década de $1970 .{ }^{(5)}$ Estudo que utilizou dados da Pesquisa Nacional de Saúde (PNS) mostrou que mais de $80,0 \%$ das mulheres utilizam algum tipo de método contraceptivo (incluindo métodos cirúrgicos), sendo o anticoncepcional oral o mais utilizado. ${ }^{(6)}$ Em outro estudo, com mulheres internadas em um hospital da mulher no município de Campinas, estado de São Paulo, Brasil, verificouse que $38,2 \%$ das pacientes iniciaram algum método contraceptivo antes da internação, com destaque para os métodos injetáveis $(51,4 \%)$ e os contraceptivos orais $(44,5 \%))^{(7)}$

A introdução dos anticoncepcionais orais tornou-se um marco na separação das relações sexuais e da reprodução, permitindo ter um melhor controle sobre uma possível gravidez, a qual poderia ser intencional ou não. ${ }^{(8)}$ Portanto, com o surgimento dos anticoncepcionais, é introduzido na história da saúde pública brasileira o planejamento 
familiar. Isso significa que a mulher, assim como seu parceiro, passa a planejar a tomada de decisão de querer ou não engravidar, como também de se prevenir de uma gravidez indesejada. ${ }^{(9)}$

Vale destacar que são escassos os dados do consumo de contraceptivos por estudantes universitárias. Neste grupo, espera-se que a utilização destes métodos seja ainda maior, sobretudo para impedir uma gravidez indesejável, mas também para regularização do ciclo menstrual e melhorar outras condições de saúde, como desregulações hormonais. Considerando o aumento do consumo de anticoncepcionais, maior exposição a relações sociais - comum no ambiente universitário, e os riscos do uso indiscriminado de anticoncepcionais, entende-se que há necessidade de traçar um perfil das estudantes universitárias sobre o uso destes medicamentos. Sendo assim, o presente estudo tem como objetivo identificar o consumo de anticoncepcionais hormonais e fatores associados entre estudantes universitárias.

\section{Métodos}

A metodologia baseou-se em um estudo de delineamento transversal, sendo a base de dados o projeto "Análise da Saúde e Hábitos de Vida dos Estudantes de Graduação da UEL" (GraduaUEL). O projeto GraduaUEL teve como objetivo analisar os hábitos de vida, questões sociais e uso de medicamentos dos estudantes da Universidade Estadual de Londrina (UEL), município de Londrina, estado do Paraná, Brasil. O GraduaUEL considerou, além dos estudantes com matrícula ativa nos cursos de graduação no primeiro semestre de 2019, também aqueles com 18 anos ou mais.

Para este estudo, além dos critérios específicos utilizados pelo GraduaUEL, também foi considerado como critério de inclusão o estudante ser do sexo feminino. A universidade apresentava, em 2019, cerca de 12.500 estudantes de graduação com 18 anos ou mais matriculados nos seus 51 cursos de graduação (50 cursos presenciais e um à distância), sendo próximo de 7.000 estudantes do sexo feminino, população deste estudo.

Os dados foram coletados a partir de um questionário eletrônico, com a utilização da plataforma Google Forms, para o qual o acesso foi feito por meio de um link criado pela equipe de pesquisadores. Assim, com a utilização dessa ferramenta foi possível uma rápida praticidade com o levantamento das respostas e a diminuição do viés de perdas das respostas. Esse instrumento apresentava inúmeras questões, incluindo dados relacionados aos hábitos de vida, dados socioeconômicos e demográficos, consumo de bebidas alcoólicas e substâncias ilícitas, violências, qualidade do sono, sintomas depressivos, consumo de medicamentos, síndrome de burnout, entre outras.

Previamente ao desenvolvimento do estudo, foi realizada validação de conteúdo com especialistas da área para adequação do instrumento. Posteriormente, o instrumento on-line foi testado com estudantes de uma universidade privada no município de Londrina e realizado um estudo piloto com estudantes de uma outra universidade pública do Paraná, localizada na região de Londrina.

Como forma de capilarizar o estudo entre os estudantes de todos os cursos de graduação da UEL, foi disponibilizado o link através das mídias sociais da universidade e na lista de e-mail dos alunos. Além disso, durante o período de coleta dos dados, professores da equipe de pesquisa e pósgraduandos dos programas de pós-graduação em Saúde Coletiva e em Ciências Farmacêuticas ou estudantes de iniciação científica ou colaboradores realizaram a divulgação presencial da pesquisa nos diversos centros de estudos e cursos de graduação da UEL.

As variáveis independentes foram: idade (em anos); orientação sexual (heterossexual; homossexual/bissexual; outra); escolaridades do pai e da mãe (analfabeto ou fundamental incompleto; fundamental completo até ensino médio completo; 
ensino superior incompleto até pós-graduação); cor (branca/amarela; preta/parda/indígena); acesso a plano de saúde privado (sim; não); indicativo de depressão (sim; não); prática de atividade física (não pratica ou pratica até uma vez na semana; pratica duas vezes ou mais na semana); satisfação corporal (muito baixa preocupação; baixa/moderada preocupação; alta preocupação); companheiro (sim; não); e Índice de Massa Corporal (IMC) (baixo peso; peso normal; sobrepeso; obesidade). ${ }^{(10)}$

Em orientação sexual, foram consideradas "outras" as estudantes que responderam "outros", “não sabem” e "prefere não responder". Para companheiro, as estudantes foram consideradas "não" quando responderam serem solteiras, porém sem namorado(a), divorciadas ou separadas; e como "sim", solteiras, mas que referiram namorado(a), casadas ou em união estável. O instrumento utilizado para identificar sintomas depressivos foi o Patient Health Questionnaire-9 (PHQ-9). ${ }^{(11)}$ Para a avaliação da satisfação corporal utilizou-se o Body Shape Questionnaire (BSQ-8). ${ }^{(12)}$

A variável dependente foi o consumo de medicamentos contraceptivos (ou anticoncepcionais - orais ou por outras vias de administração). Além da identificação do uso destes medicamentos, também foram avaliados o tempo de utilização e o responsável pela prescrição (médico ou outro, incluindo automedicação). Para a identificação e a classificação dos medicamentos anticoncepcionais foi utilizada a classificação Anatomic Therapeutic Chemical (ATC), da Organização Mundial da Saúde (OMS), ${ }^{(13)}$ com a utilização dos seguintes códigos: G03AA07; G03AA09; G03AA10; G03AA12; G03AA14; G03AA15; G03AA16; G03AB08; G03AC08; G03AC09; G03AC01; G03CA53; G03DB08; G03FA01; e G03HB01.

Após a finalização da coleta de dados, estes foram exportados para uma planilha do software Microsoft ${ }^{\circledR}$ Excel $^{\circledR}$, a qual foi conferida para identificar possíveis inconsistências, que foram corrigidas. A análise estatística foi realizada por meio de análises descritivas e de associação, bi e multivariadas. Para tal foi utilizado o software IBM $^{\circledR}$ SPSS $^{\circledR}$, versão 19.0, com cálculo da Razão de Prevalência utilizando-se a Regressão de Poisson com variância robusta. Foi feita, inicialmente, uma análise bruta e, em seguida, uma análise ajustada, na qual foram inseridas todas as variáveis independentes em um mesmo modelo.

O GraduaUEL foi aprovado pelo Comitê de Ética em Pesquisa (CEP) da Universidade Estadual de Londrina, CAAE sob n ${ }^{\circ}$ 04456818.0.0000.5231. Todos os participantes do GraduaUEL foram devidamente esclarecidos sobre os objetivos do estudo e assinaram o Termo de Consentimento Livre e Esclarecido (TCLE), de forma eletrônica, quando concordaram em participar da pesquisa.

\section{Resultados}

Foram observados 3.525 registros ao final da coleta de dados. Após conferência por dupla de pesquisadores, foram excluídos 82 registros duplicados ou triplicados e 1.208 questionários por estudantes que não atendiam aos critérios de inclusão (inclusive sexo masculino), resultando em 2.235. Desses, foram consideradas perdas: 12 por não informarem a idade e dois por estarem majoritariamente não respondidos ou com inconsistências no preenchimento. Assim, foram considerados válidos 2.221 questionários respondidos por estudantes do sexo feminino.

Das respondentes $(\mathrm{N}=2.221)$, a maioria relatou ser heterossexual $(76,3 \%)$, com quase metade das mães apresentando ensino superior incompleto e mais, e maioria dos pais com ensino fundamental completo até ensino médio. A grande parte das estudantes era da cor branca/amarela $(75,8 \%)$, foi classificada com indicativo de depressão $(77,4 \%)$ e não praticava ou praticava atividade física, no máximo, uma vez na semana $(71,6 \%)$. Outras informações sobre as características das estudantes podem ser visualizadas na Tabela 1 . 
Tabela 1 - Distribuição das estudantes universitárias segundo características sociodemográficas, de saúde e hábitos de vida. GraduaUEL, 2019. ( $\mathrm{N}=2.221)$.

\begin{tabular}{|c|c|c|c|}
\hline \multirow[b]{2}{*}{ Características das estudantes } & \multirow{2}{*}{$\begin{array}{c}\text { Total } \\
\text { N (\%) }\end{array}$} & \multicolumn{2}{|c|}{ Consumo de anticoncepcionais } \\
\hline & & $\begin{array}{c}\text { Sim } \\
\text { N (\%) }\end{array}$ & $\begin{array}{c}\text { Não } \\
\text { N (\%) }\end{array}$ \\
\hline \multicolumn{4}{|l|}{ Orientação sexual } \\
\hline Heterossexual & $1694(76,3)$ & $252(14,9)$ & $1442(85,1)$ \\
\hline Homossexual/bissexual & $83(3,7)$ & $11(13,3)$ & $72(86,7)$ \\
\hline Outra & $444(20,0)$ & $35(7,9)$ & $409(92,1)$ \\
\hline \multicolumn{4}{|l|}{ Cor } \\
\hline Branca/amarela & $1683(75,8)$ & $246(14,6)$ & $1437(85,4)$ \\
\hline Preta/parda/indígena & $538(24,2)$ & $52(9,7)$ & $486(90,3)$ \\
\hline \multicolumn{4}{|l|}{ Companheiro } \\
\hline Não & $1115(50,2)$ & $120(10,8)$ & $995(89,2)$ \\
\hline Sim & $1106(49,8)$ & $178(16,09)$ & $928(83,9)$ \\
\hline \multicolumn{4}{|l|}{ Escolaridade da mãe } \\
\hline Analfabeta/fundamental incompleto & $287(12,9)$ & $27(9,4)$ & $260(90,6)$ \\
\hline Fundamental completo até o ensino médio completo & $871(39,2)$ & $107(12,3)$ & $764(87,7)$ \\
\hline Superior incompleto ou mais & $1048(47,2)$ & $162(15,5)$ & $886(84,5)$ \\
\hline \multicolumn{4}{|l|}{ Escolaridade do pai } \\
\hline Analfabeto/fundamental incompleto & $375(16,9)$ & $33(8,8)$ & $342(91,2)$ \\
\hline Fundamental completo até o ensino médio completo & $925(41,6)$ & $119(12,9)$ & $806(87,1)$ \\
\hline Superior incompleto ou mais & $831(37,4)$ & $136(16,4)$ & $695(83,6)$ \\
\hline \multicolumn{4}{|l|}{ Acesso a plano de saúde } \\
\hline Não & $1194(53,8)$ & $134(11,2)$ & $1060(88,8)$ \\
\hline Sim & $1025(46,2)$ & $164(16,0)$ & $861(84,0)$ \\
\hline \multicolumn{4}{|l|}{ Indicativo de depressão } \\
\hline Não & $448(20,7)$ & $61(13,6)$ & $387(86,4)$ \\
\hline $\operatorname{Sim}$ & $1720(77,4)$ & $230(13,4)$ & $1490(86,6)$ \\
\hline \multicolumn{4}{|l|}{ Prática de atividade física } \\
\hline Não ou até 1 vez na semana & $1590(71,6)$ & $200(12,6)$ & $1390(87,4)$ \\
\hline Duas vezes ou mais na semana & $631(28,4)$ & $98(15,5)$ & $533(84,5)$ \\
\hline \multicolumn{4}{|l|}{ Satisfação corporal } \\
\hline Muito baixa preocupação & $568(25,6)$ & $77(13,6)$ & $491(86,4)$ \\
\hline Baixa/moderada preocupação & $1010(45,5)$ & $147(14,6)$ & $863(85,4)$ \\
\hline Alta preocupação & $597(26,9)$ & $70(11,7)$ & $527(88,3)$ \\
\hline \multicolumn{4}{|l|}{ Índice de Massa Corporal (IMC) } \\
\hline Baixo peso & $220(9,9)$ & $33(15,0)$ & $187(85,0)$ \\
\hline Peso normal & $1376(62,2)$ & $196(14,2)$ & $1180(85,8)$ \\
\hline Sobrepeso & $417(18,8)$ & $45(10,8)$ & $372(83,2)$ \\
\hline Obesidade & $200(9,0)$ & $24(12,0)$ & $176(88,0)$ \\
\hline
\end{tabular}

Fonte: os autores. 
Das 2.221 estudantes avaliadas, $298(13,0 \%)$ indicaram utilizar algum medicamento contraceptivo. Destes, a maioria foi indicada por médicos $(92,6 \%)$. O tempo de uso superior a seis meses foi o mais prevalente $(77,9 \%)$ (Tabela 2 ). Dentre os diversos contraceptivos, os mais utilizados pelas estudantes estão listados na Tabela 3, sendo os mais frequentes princípios ativos a drospirenona e etinilestradiol, seguidos da ciproterona e estrogênio, e gestodeno e etinilestradiol.

Tabela 2 - Caracterização do uso de medicamentos contraceptivos segundo indicação e tempo de utilização. GraduaUEL, 2019. (N=298).

\begin{tabular}{lc}
\hline Medicamentos contraceptivos hormonais & N $(\mathbf{\%})$ \\
\hline Indicação do contraceptivo* & $276(92,6)$ \\
Médicos & $8(2,7)$ \\
Uso por conta própria & $7(2,3)$ \\
Amigos/familiares & $3(1,0)$ \\
Farmacêuticos/atendente de farmácia & $1(0,4)$ \\
Outro & \\
Tempo de uso do contraceptivo* & $16(5,4)$ \\
Menos de 3 meses & $25(8,4)$ \\
3 meses a 6 meses & $21(7,0)$ \\
7 meses a 12 meses & $232(77,9)$ \\
Mais de 12 meses
\end{tabular}

*O total é inferior a 298, pois algumas estudantes não responderam a este questionamento.

Fonte: os autores.

Tabela 3 - Distribuição dos medicamentos contraceptivos utilizados segundo substância química (denominação genérica). GraduaUEL, 2019. ( $\mathrm{N}=298)$.

\begin{tabular}{lc}
\hline Princípio ativo - Código ATC & N (\%) \\
\hline Drospirenona e etinilestradiol - G03AA12 & $111(37,2)$ \\
Ciproterona e estrogênio - G03HB01 & $54(18,1)$ \\
Gestodeno e etinilestradiol - G03AA10 & $40(13,4)$ \\
Nomegestrol e estradiol - G03AA14 & $19(6,4)$ \\
Levonorgestrel e etinilestradiol - G03AA07 & $15(5,0)$ \\
Desogestrel e etinilestradiol - G03AA09 & $13(4,4)$ \\
Desogestrel - G03AC09 & $11(3,7)$ \\
Dienogeste e estradiol - G03AB08 & $10(3,4)$ \\
Clormadinona e etinilestradiol - G03AA15 & $9(3,0)$ \\
Dienogeste - G03DB08 & $8(2,7)$ \\
Estradiol e associações - G03CA53 & $2(0,7)$ \\
Dienogeste e etinilestradiol - G03AA16 & $1(0,3)$ \\
Etonogestrel - G03AC08 & $1(0,3)$ \\
Noretisterona - G03AC01 & $1(0,3)$ \\
Noretisterona e estrogênio - G03FA01 & $1(0,3)$ \\
Não identificado & $2(0,7)$ \\
\hline
\end{tabular}

Fonte: os autores. 
$\mathrm{Na}$ Tabela 4, na análise bruta observa-se que o uso de contraceptivos foi maior por estudantes heterossexuais, com mães e/ou pais que tinham ensino, de cor branca/amarela, com acesso a plano de saúde, que relataram praticar atividade física duas vezes ou mais na semana e que declararam possuir companheiro. $\mathrm{Na}$ análise ajustada apenas as estudantes heterossexuais mantiveram associação com o consumo de contraceptivos (RP: 1,865; IC 95\%: 1,308-2,659).

Tabela 4 - Associação entre as variáveis independentes e o consumo de medicamentos contraceptivos. GraduaUEL, 2019.

\begin{tabular}{|c|c|c|c|c|}
\hline \multirow[b]{2}{*}{ Variável independente } & \multicolumn{4}{|c|}{ Consumo de anticoncepcionais } \\
\hline & $\begin{array}{l}\text { RP bruta } \\
\text { (IC 95\%) }\end{array}$ & Valor de $\mathbf{p}$ & $\begin{array}{l}\text { RP ajustada* } \\
\text { (IC 95\%) }\end{array}$ & Valor de $p$ \\
\hline Idade & $0,930(0,945-1,015)$ & 0,263 & $0,980(0,945-1,015)$ & 0,263 \\
\hline \multicolumn{5}{|l|}{ Orientação sexual } \\
\hline Heterossexuais & $1,849(1,306-2,618)$ & 0,001 & $1,865(1,308-2,659)$ & 0,001 \\
\hline Homossexual/bissexual & $1,534(0,767-3,068)$ & 0,226 & $1,689(0,846-3,372)$ & 0,138 \\
\hline Outra & 1,00 & 1,00 & 1,00 & \\
\hline \multicolumn{5}{|l|}{ Escolaridade da mãe } \\
\hline Analfabeta/fundamental incompleto & 1,00 & & 1,00 & \\
\hline $\begin{array}{l}\text { Fundamental completo } \\
\text { até o ensino médio completo }\end{array}$ & $1,354(0,887-2,067)$ & 0,160 & $1,074(0,691-1,672)$ & 0,750 \\
\hline Superior incompleto ou mais & $1,684(1,120-2,533)$ & 0,012 & $1,154(0,730-1,824)$ & 0,539 \\
\hline \multicolumn{5}{|l|}{ Escolaridade do pai } \\
\hline Analfabeto/fundamental incompleto & 1,00 & & 1,00 & \\
\hline $\begin{array}{l}\text { Fundamental completo } \\
\text { até o ensino médio completo }\end{array}$ & $1,446(0,997-2,098)$ & 0,052 & $1,198(0,810-1,772)$ & 0,366 \\
\hline Superior incompleto ou mais & $1,815(1,258-2,617)$ & 0,001 & $1,303(0,856-1,984)$ & 0,217 \\
\hline \multicolumn{5}{|l|}{ Cor } \\
\hline Branca/amarela & $1,545(1,139-2,096)$ & 0,005 & $1,317(0,964-1,800)$ & 0,084 \\
\hline Preta/parda/indígena & 1,00 & & 1,00 & \\
\hline \multicolumn{5}{|l|}{ Acesso a plano de saúde } \\
\hline Não & 1,00 & & 1,00 & \\
\hline $\operatorname{Sim}$ & $1,385(1,111-1,727)$ & 0,004 & $1,189(0,930-1,519)$ & 0,167 \\
\hline \multicolumn{5}{|l|}{ Indicativo de depressão } \\
\hline Não & 1,00 & & 1,00 & \\
\hline $\operatorname{Sim}$ & $1,034(0,793-1,348)$ & 0,807 & $0,900(0,681-1,189)$ & 0,458 \\
\hline \multicolumn{5}{|l|}{ Prática de atividade física } \\
\hline Não ou até 1 vez na semana & 1,00 & & 1,00 & \\
\hline Duas vezes ou mais na semana & $1,309(1,041-1,646)$ & 0,021 & $1,210(0,959-1,526)$ & 0,108 \\
\hline \multicolumn{5}{|l|}{ Satisfação corporal } \\
\hline Muito baixa preocupação & $1,187(0,870-1,621)$ & 0,280 & $1,208(0,855-1,708)$ & 0,284 \\
\hline Baixa/moderada preocupação & $1,217(0,923-1,607)$ & 0,164 & $1,207(0,907-1,605)$ & 0,197 \\
\hline Alta preocupação & 1,00 & & 1,00 & \\
\hline
\end{tabular}




\section{Continuação}

$\begin{array}{lcccc}\text { Companheiro } & & & & \\ \text { Não } & 1,330(0,669-2,644) & 0,415 & 1,045(0,471-2,317) & 0,914 \\ \text { Sim } & 2,113(1,072-4,168) & 0,031 & 1,581(0,719-3,474) & 0,255 \\ \text { Índice de Massa Corporal (IMC) } & & & & \\ \quad \text { Baixo peso } & 1,392(0,804-2,408) & 0,237 & 1,018(0,575-1,804) & 0,950 \\ \text { Peso normal } & 1,411(0,894-2,228) & 0,139 & 1,054(0,639-1,629) & 0,934 \\ \text { Sobrepeso } & 1,009(0,596-1,707) & 0,975 & 0,870(0,516-1,468) & 0,603 \\ \text { Obesidade } & 1,00 & & 1,00 & \end{array}$

*No modelo ajustado foram incluídas todas as variáveis independentes.

Fonte: os autores.

\section{Discussão}

De acordo com os resultados apresentados, há maior consumo de contraceptivos hormonais por mulheres brancas ou amarelas, que relataram serem heterossexuais, que apresentavam pais com maior escolaridade, que referiram possuir plano privado de saúde e companheiro, e que praticavam atividade física pelo menos duas vezes na semana. Entretanto, apenas o fato de serem heterossexuais mostrou-se associação significativa após a análise ajustada.

Apesar de os resultados demonstrarem que a maioria das mulheres faz uso de contraceptivos por indicação médica, ainda tem se mostrado um papel relevante a prática da automedicação $(7,4 \%)$, mesmo que em parte tenha havido indicação por profissional farmacêutico (1,0\%). Todavia, deve-se destacar que contraceptivos hormonais são medicamentos sob prescrição médica, não podendo ser indicados/prescritos por farmacêuticos. Estudo que analisou os riscos e benefícios dos contraceptivos demonstrou que o uso por indicação de conhecidos é maior do que aqueles que procuram indicação por profissionais da saúde, exceto os médicos. ${ }^{(14)}$

A automedicação é considerada comum na sociedade, já que há maior facilidade de acesso rápido ao medicamento, com menor gasto de tempo e dinheiro, pois consulta médica privada envolve custos financeiros, assim como consumo pela influência do marketing e da indústria farmacêutica. ${ }^{(15)}$
No entanto, essa prática apresenta riscos, tais como efeitos adversos e interações medicamentosas. Dados do Sistema Nacional de Informações TóxicoFarmacológicas (Sinitox) apontam que dentre os agentes tóxicos que mais causaram intoxicações em 2017, 26,8\% são medicamentos. ${ }^{(16)}$

Estudo demonstrou que os anticoncepcionais combinados geram maiores riscos trombóticos, ao se comparar com pessoas que não utilizam nenhum anticoncepcional. Em adição, o uso de anticoncepcionais combinados de $30 \mu \mathrm{g}-35 \mu \mathrm{g}$ (etinilestradiol e gestodeno, desogestrel, acetato de ciproterona ou drospirenona) apresentou riscos de 50\%-80\% maiores do que os anticoncepcionais combinados com levonorgestrel. ${ }^{(17)}$ Logo, usuárias e principalmente aquelas que apresentam comorbidades que usam anticoncepcionais orais combinados como os citados anteriormente, que possivelmente poderiam ser indicados por amigos e familiares, podem apresentar maiores riscos de eventos trombóticos. Vale ressaltar que este estudo identificou que há maior prevalência de uso dos princípios ativos drospirenona e etinilestradiol, ciproterona e estrogênio, e gestodeno e etinilestradiol.

Os resultados encontrados mostraram que as estudantes com pais com menor escolaridade e que não possuíam plano de saúde privado são as que menos utilizam contraceptivos hormonais. Estudos demonstram que quanto maior o nível de escolaridade, mesmo que dos pais, maiores são o nível socioeconômico e a adesão a plano privado de saúde, 
assim apresentando melhores conhecimentos sobre métodos contraceptivos e fundamentalmente maior acesso aos mesmos. Ainda é importante destacar que mulheres que apresentam condições socioeconômicas baixas tendem a usar o contraceptivo hormonal de forma inadequada, ${ }^{(18)}$ além de que, quando associado à baixa escolaridade, há mais chances de ocorrer uma gravidez indesejada. ${ }^{(19)}$

No que se refere ao fato de as mulheres com companheiro utilizarem mais os contraceptivos, é notado que quando comparado com os outros métodos disponíveis, os contraceptivos hormonais são os que mais prevalecem. Ainda, por possuírem parceiros fixos, utilizam menos a dupla proteção (anticoncepcional e camisinha) do que as sem parceiros. ${ }^{(6)}$ As que não têm parceiros fixos tendem a utilizar mais métodos de barreira, pois além da proteção contra uma gravidez indesejável, também garante proteção contra as infecções sexualmente transmissíveis (ISTs). ${ }^{(20)}$

Com relação ao uso dos contraceptivos ser menor entre as usuárias da comunidade LGBTQI+ do que pelas heterossexuais, possivelmente decorre de as primeiras terem maiores dificuldades quanto ao uso de serviços de saúde, uma vez que ainda há discriminação e a invisibilidade da sociedade por sua sexualidade. Assim, por terem poucas informações sobre estes medicamentos, há menor utilização e precariedade do seu conhecimento. ${ }^{(21-22)}$ Além desses motivos, pode-se notar que essas usuárias sentem dificuldades em questionar ou buscar ajuda de um profissional de saúde, pois percebem a diferença da percepção quando são tratadas diante das mulheres heterossexuais, deixando-as cada vez mais receosas e com medo de buscar ajuda. ${ }^{(23)}$

De forma adicional, o uso de contraceptivo hormonal identificado neste estudo foi maior no grupo heterossexual do que no grupo homossexual ou bissexual, mas principalmente entre aqueles que referiram outra orientação sexual ou preferiram não responder à questão. Pesquisas em geral mostram que as bissexuais utilizam mais contraceptivos reversíveis de longa duração (dispositivo intrauterino - DIU, por exemplo) do que as homossexuais, e que há necessidade de atendimento mais amplo nos serviços clínicos e na forma do lidar com as pacientes. Assim, sugere-se que os profissionais de saúde conduzam seus pacientes para que tenham maior alcance na conscientização para esses métodos, além de que, mulheres de minorias sexuais apresentam maior probabilidade de não realizarem cuidados ginecológicos preventivos. ${ }^{(24-26)}$ Deve-se destacar, ainda, que as mulheres homossexuais, importante grupo da comunidade LGBTQI+, por não terem relações heterossexuais, não utilizam contraceptivos para fins de prevenção da gravidez, e sim para regulação hormonal ou menstrual, o que justifica também o menor consumo destes medicamentos por este segmento.

O presente estudo também mostrou que houve maior consumo de anticoncepcionais por parte de mulheres brancas/amarelas do que para as mulheres pretas/pardas/indígenas. Isso sugere que pode haver desigualdade racial no acesso a estes medicamentos. Desde os tempos passados as populações preta e indígena têm sido discriminadas, devido à exploração e à escravização, além de ser muito frequente o racismo institucional, com diferença quanto ao acesso aos bens e serviços públicos e de saúde, discriminações pelo comportamento, negligências e estereótipos. ${ }^{(27-28)}$

Além dessa explicação, entre o período de 1996-2006 as mulheres pobres e negras apresentaram maior prevalência de gravidez não planejada quando comparadas com as mulheres brancas. ${ }^{(9)}$ Acresce-se que, conforme a análise feita por Cruz, Carvalho e Irffi (2016), ${ }^{(29)}$ a probabilidade de a mulher branca ter uma gravidez precoce é menor, comparada com as mulheres pretas, pardas e indígenas. Logo, nos tempos atuais, a mulher preta tem sentido desigualdades quanto aos seus direitos reprodutivos, assim como na oferta dos serviços de saúde e nos determinantes sociais, que, de certa forma, afetam sua saúde reprodutiva. ${ }^{(30)}$

Ademais, estudo com dados da Pesquisa Nacional de Saúde (PNS) identificou que mulheres brancas utilizam a dupla proteção (camisinha e anticoncepcional oral), já as mulheres pretas e 
indígenas usam mais frequentemente métodos de barreira, como a camisinha, pois sua utilização é prática, não precisa de prescrição e também é fácil de se adquirir gratuitamente para além do próprio Sistema Único de Saúde (SUS). ${ }^{(6)}$

Quanto à associação da prática de exercícios físicos ao uso de contraceptivo hormonal, há poucas pesquisas que possam relacionar a sua associação. Entretanto, estudo de caso mostrou que a prática de exercícios físicos associada ao uso de contraceptivo oral combinado apresenta melhora nos perfis lipídico e inflamatório e no IMC. Essa melhora é gerada pela diminuição ao LDL, o que significa que essa lipoproteína está relacionada ao processo inflamatório devido à aterogênese que ocasiona problemas cardíacos, já que a prática de exercícios físicos gera maior consumo de energia, gerado pela quebra dos ácidos graxos e glicerol através dos triglicerídeos. ${ }^{(31)}$ Dessa maneira, podese inferir que a prática de atividade física pode ser uma estratégia para minimizar o risco inerente ao uso de contraceptivos hormonais.

Algumas limitações da presente investigação devem ser destacadas: 1) a coleta de dados foi realizada de forma on-line e o preenchimento de um questionário eletrônico impossibilita a explicação detalhada no momento da pesquisa; 2) por ser um instrumento autopreenchido, as informações sobre os medicamentos podem estar sujeitas ao viés de memória; e 3) a taxa de resposta para esta pesquisa apresentou-se menor que a observada em outros estudos com questionários eletrônicos, ${ }^{(32)}$ o que pode comprometer a validade interna do estudo. Todavia, o tamanho da amostra do presente estudo é superior ao de outras pesquisas com estudantes de graduação e a coleta on-line possibilitou captar dados de diversas áreas acadêmicas, viabilizando uma amostra com uma população mais representativa da comunidade universitária.

Diante dos fatos acima, percebe-se que há necessidade de conversar, divulgar e preparar os profissionais da saúde sobre a utilização de contraceptivos hormonais, além de relatar seus efeitos adversos. Ainda, que questões sociais devem ser consideradas no processo de uso dos contraceptivos, para combater a discriminação e as desigualdades social e econômica quanto ao acesso e uso destes medicamentos. Como o consumo de contraceptivos foi maior nas estudantes com acesso a plano de saúde, da cor branca ou amarela e que referiram pais com maior escolaridade e proxies de maior nível socioeconômico, políticas públicas para facilitar o acesso às medidas contraceptivas, em especial os anticoncepcionais hormonais, são fundamentais para garantir equidade no processo de planejamento familiar, gravidezes indesejáveis e acesso às terapias hormonais necessárias às jovens com distúrbios passíveis de tratamento com contraceptivos hormonais.

\section{Referências}

1 Instituto Brasileiro de Geografia e Estatística (IBGE). Idosos indicam caminhos para uma melhor idade. [Internet]. 2019 Mar 03. [citado 2021 maio 10]. Disponível em: https://censo 2021.ibge.gov.br/2012-agencia-de-noticias/ noticias/24036-idosos-indicam-caminhos-parauma-melhoridade.html

2 Instituto Brasileiro de Geografia e Estatística (IBGE). Brasil em síntese: população, taxas de fecundidade total. [Internet]. 2013. [citado 2021 maio 10]. Disponível em: https://brasilemsintese. ibge.gov.br/populacao/taxas-de-fecundidadetotal.html

3 Núcleo Brasileiro de Estágios (NUBE). Pesquisa revela se os jovens querem ter filhos. [Internet]. 2018. [citado 2021 maio 3]. Disponível em: https://www.nube.com.br/blog/2018/08/08/ pesquisa-revela-se-os-jovens-querem-ter-filhos

4 Puig-Barrachina V, Domínguez-Berjón MF, Rodríguez-Sanz M, Martín U, Luque MA, Ruiz $\mathrm{M}$, et al. Decline in fertility induced by economic recession in Spain. Gac Sanit. 2020 May-Jun; 34:238-44. doi: 10.1016/j.gaceta.2019.05.011.

5 Organização das Nações Unidas (ONU). Trends in contraceptive use worldwide 2015. [Internet]. New York: United Nations; 2015. [cited 2021 may 16]. Disponível em: https://www.un.org/ 
6 Trindade RE, Siqueira BB, Paula TF, FelisbinoMendes MS. Uso de contracepção e desigualdades do planejamento reprodutivo das mulheres Brasileiras. Ciênc Saúde Colet. [Internet]. 2021. [citado 2021 maio 10]. Disponível em: http://www.cienciaesaudecoletiva.com.br/ artigos/uso-de-contracepcao-e-desigualdadesdo-planejamento-reprodutivo-das-mulheresbrasileiras $/ 17372$ ? id $=17372$

7 Veiga-Junior NN, Cavalari CA, Eugeni C, Kajiura BD, Stefano N, Baccaro LF. Postabortion contraception before hospital discharge after installation of a surveillance network in Brazil. Int J Gynecol Obstet. 2020 Aug;150(2):200-05. doi: 10.1002/ijgo.13170.

8 United Nations Population Fund (UNFPA). Situação da População Mundial 2019: um trabalho inacabado: a busca por direitos e escolhas para todos e todas. [Internet]. UNFPA; 2019. [citado 2021 out 14]. Disponível em: https://brazil.unfpa.org/pt-br/topics/swop2019

9 Ministério da Saúde (BR). Pesquisa Nacional de Demografia e Saúde da Criança e da Mulher - PNDS 2006: dimensões do Processo Reprodutivo e da Saúde da Criança. [Internet]. Brasília: Ministério da Saúde; 2009. [citado 2021 maio 16]. Disponível em: http://bvsms. saude.gov.br/bvs/publicacoes/pnds_crianca mulher.pdf

10 World Health Organization (WHO). Physical status: the use and interpretation of anthropometry. Report of a WHO Expert Committee Geneva: WHO; 1995.

11 Santos IS, Tavares BF, Munhoz TN, Almeida LSP, Silva NTB, Tams BD, et al. Sensibilidade e especificidade do Patient Health Questionnaire-9 (PHQ-9) entre adultos da população geral. Cad Saúde Pública. 2013 Ago; 29(8):1533-43. doi: 10.1590/0102-311X001 44612.

12 Teixeira PA, Locatelli KMM, Silva WR. Preocupação com a forma corporal e avaliação nutricional em estudantes de uma universidade privada. RBONE: Rev Bras Obes Nutr Emagrec. 2018 Jan-Dez; 12(75):883-91.
13 World Health Organization (WHO). WHO Collaboration Centre for Drug Statistics Methodology. ATC/DDD Index 2019. [Internet] 2019. [cited 2021 may 11]. Disponível em: https://www.whocc.no/atc_ddd_index/

14 Pinto LFA, Rodovalho-Callegari FV, Carbol M. Conhecimento de universitárias sobre os riscos e benefícios associados aos contraceptivos orais combinados. Rev Med. 2020 Set-Out; 99(5):42331. doi: 10.11606/issn.1679-9836.v99i5p423-431.

15 Paula CCS, Campos RBF, Souza MCRF. Uso irracional de medicamentos: uma perspectiva cultural. Braz J Dev. 2021 Mar; 7(3):2166076. doi: 10.34117/bjdv7n3-060.

16 Sistema de Informações Tóxico-Farmacológicas (SINITOX). Dados de Intoxicação. [Internet]. 2017. [citado 2021 maio 08]. Disponível em: https://sinitox.icict.fiocruz.br/sites/sinitox. icict.fiocruz.br/files//Brasil8_1.pdf

17 Bastos M, Stegeman BH, Rosendaal FR, Vlieg AVH, Helmerhorst FM, Stijnen T, et al. Combined oral contraceptives: venous thrombosis. Cochrane Database Syst Rev. 2014 Mar; (3):CD010813. doi: 10.1002/14651858. CD010813.pub2.

18 Corrêa DAS. Uso de contraceptivos orais entre mulheres de 18 a 49 anos: inquérito populacional telefônico [dissertação]. Belo Horizonte (MG): Escola de Enfermagem, Universidade Federal de Minas Gerais; 2012.

19 Iseyemi A, Zhao Q, McNicholas C, Peipert JF. Socioeconomic status as a risk factor for unintended pregnancy in the contraceptive CHOICE project. Obstet Gynecol. 2017 Sep; 130(3):60915. doi: 10.1097/AOG.0000000000002189.

20 Gutierrez EB, Pinto VM, Basso CR, Spiassi AL, Lopes MEBR, Barros CRS. Fatores associados ao uso de preservativo em jovens - inquérito de base populacional. Rev Bras Epidemiol. 2019; 22:e190034. doi: 10.1590/1980-549720190034.

21 Sousa AJM, Barros AL. Homosexual women's health: approaches on a necessary theme. Rev Enferm UFPI. 2020; 9:e11546. doi: 10.26694/ reufpi.v9i0.11546. 
22 Paiva EMC, Dias JF, Calheiros AP, Nogueira $\mathrm{DA}$, et al. Uso de métodos contraceptivos entre acadêmicos da área da saúde. Semin Ciênc Biol Saúde. 2020 Jun-Dez; 41(Supl.2):331-40. doi: 10.5433/1679-0367.2020v41n2Suplp331.

23 Rodrigues DLM. O rastreio e a prevenção das ISTs em mulheres lésbicas e bissexuais: revisão integrativa [trabalho de conclusão de curso]. Cajazeiras (PB): Universidade Federal de Campina Grande; 2019.

24 Charlton BM, Janiak E, Gaskins AJ, DiVasta $\mathrm{AD}$, Jones RK, Missmer SA, et al. Contraceptive use by women across different sexual orientation groups. Contraception. 2019 Sep; 100(3):202-08. doi: 10.1016/j.contraception. 2019.05.002.

25 Everett BG, Sanders JN, Myers K, Geist C, Turok DK. One in three: challenging heteronormative assumptions in family planning health centers. Contraception. 2018 Oct; 98(4):27074. doi: 10.1016/j.contraception.2018.06.007.

26 McCauley HL, Dick RN, Tancredi DJ, Goldstein S, Backburn S, Silverman JG, et al. Differences by sexual minority status in relationship abuse and sexual and reproductive health among adolescent females. J Adolesc Health. 2014 Nov; 55(5):652-58. doi: 10.1016/j.jadohealth. 2014.04.020.

27 Kalckmann S, Santos CG, Batista LE, Cruz VM. Racismo institucional: um desafio para a equidade no SUS?. Saúde Soc. 2007 Ago; 16(2):146-55. doi: 10.1590/S0104-12902007 000200014 .

28 Loduvico GO, Martins MML, Rocha TIU, Terra MF, Pigozi PL. Racismo institucional: percepção sobre a discriminação racial nos serviços de saúde. Arq Méd Hosp Fac Cienc Med Santa Casa São Paulo. 2021; 66:e008. doi: 10.26432/1809-3019.2021.66.008.

29 Cruz MS, Carvalho FJV, Irffi GD. Perfil socioeconômico, demográfico, cultural, regional e comportamental da gravidez na adolescência no Brasil. Planej Polít Públicas. 2016 Jan-Jun; 46:243-66.
30 Prietsch SOM, González-Chica DA, Cesar JA, Mendoza-Sassi RA. Gravidez não planejada no extremo Sul do Brasil: prevalência e fatores associados. Cad. Saúde Pública. 2011 Out; 27(10):1906-16. doi: 10.1590/S0102311X2011001000004.

31 Oliveira LA, Silva VC, Soares TA, Rosa EA, Petto J. Exercício e contraceptivo: influência no perfil lipídico e inflamatório. Rev Bras Fisiol Exerc. 2018; 17(1);47-50. doi: 10.33233/rbfe. v17i1.2368.

32 Blumenberg C, Barros AJD. Response rate differences between web and alternative data collection methods for public health research: a systematic review of the literature. Int J Public Health. 2018 Jul; 63(6):765-73. doi: 10.1007/ s00038-018-1108-4. 